\title{
Myasthenic syndrome: effect of choline, plasmapheresis and tests for circulating factor
}

\author{
HENRYK KRANZ, DAVID J CADDY, ANDREW M WILLIAMS, AND \\ WANSOON GAY
}

\begin{abstract}
From the Department of Neurology and Clinical Neurophysiology, Alfred Hospital and Department of Pharmacology, Melbourne University, Melbourne, Australia
\end{abstract}

SUMMARY In a patient with myasthenic syndrome neuro-muscular transmission was characterised by depression and facilitation. The relative extent of these two processes varied between muscles, and in the one muscle with time. Guanidine $\mathrm{HCl}$ treatment corrected the electrophysiological defect. Oral choline increased muscle action potential amplitude in response to single shocks. Intravenous choline produced features indicating cholinergic autonomic stimulation. Pimozide and plasmapheresis had no effect. Animal in-vivo and in-vitro studies performed to detect a circulating factor which interferes with neuro-muscular transmission were negative.

The myasthenic syndrome (MYS) is a well defined clinical entity. ${ }^{1}$ There remain a number of unresolved aspects, including a lack of knowledge of the cause and mechanism of the disorder. We have quantified certain parameters of neuromuscular transmission in one patient with MYS in order to further define the pathophysiology of this condition. The transmission defect in MYS has two components, characterised by depression and facilitation. A variation in magnitude of these components with time, and at different neuro-muscular junctions, is demonstrated. This could explain the apparent concurrent presence of transmission features of myasthenia gravis (MG) and MYS which have been reported in scme patients. ${ }^{2-4}$

The effect of certain pharmacological agents, in particular choline, was assessed, and the influence of plasmapheresis noted. The patient's serum was tested for its effect on neuro-muscular transmission in animal in-vivo and in-vitro preparations.

\section{Patients and methods}

A 56 year old woman presented with several months history of proximal weakness. Thyroid function tests were in the toxic range: T3 7.5

Address for reprint requests: Dr H Kran7, Department of Neurology, Alfred Hospital, Melbourne, 3181, Australia.

Accepted 7 February 1980 $\mathrm{nmol} / \mathrm{l}(1 \cdot 0-2 \cdot 7)$, T4 $250 \mathrm{nmol} / 1(60-145)$, FTI $360(50-140)$, T3 resin uptake $144 \%(80-110)$, ${ }^{131}$ I thyroid uptake $20 \%$ at four hours. She was treated with carbimazole $60 \mathrm{mg} /$ day and euthyroid status was reached after six weeks. Weakness persisted and three months later electrophysiological studies showed the characteristic features of MYS. Serum electrolytes including calcium, magnesium, manganese and cobalt were normal. Anti-acetylcholine receptor antibody test was negative. Repeated screening for an occult malignancy was negative.

Neuromuscular transmission was studied by recording compound action potential amplitude $(\mathrm{CAPa})$ with superficial electrodes in response to supramaximal nerve stimulation. This was done in thenar, extensor digitorum brevis (EDB) and quadriceps muscles. Change in CAPa following nerve stimulation or exercise was expressed and plotted as a percentage change [ $(100 \times$ test CAPa $/$ control $\mathrm{CAPa}-100 \%$ ]. Care was taken to have similar temperature at test sites.

Ir-vivo tests Edrophonium $\mathrm{HCl}$ was administered intravenously in a divided dose of $2 \mathrm{mg}$ and $8 \mathrm{mg}$ three minutes apart, preceded by atropine $0.6 \mathrm{mg}$ intravenously. Choline was given intravenously as choline bitartrate. Calculated as the equivalent dose of choline $\mathrm{Cl}$ it was infused in normal saline at a rate of $27 \mathrm{mg} / \mathrm{min}$ over 70 min for a total dose of $38 \mathrm{mg} / \mathrm{kg}$. Choline $\mathrm{Cl}$ was also given orally in a dose of $210 \mathrm{mg} / \mathrm{kg} / \mathrm{day}$ for four weeks. Pimozide was given orally in a 
dose of $6 \mathrm{mg} /$ day for three weeks. Guanidine $\mathrm{HCl}$ was taken in a dose of $20 \mathrm{mg} / \mathrm{kg} /$ day orally over five months.

Continuous-flow plasma exchange was performed (Model 30 Haemonetics) with a total of six exchanges spaced 2, 5, 7, 9, and seven days apart. Mean exchange volume was $2520 \mathrm{ml}$, resulting in a drop in serum Ig A,G and $M$ of $60 \%$.

The effect of the patient's serum on the sciatic nerve-tibialis anterior muscle preparation ${ }^{5}$ of the cat was assessed. Twitch responses produced by supramaximal stimulation of the sciatic nerve were monitored. This was done under general anaesthesia with and without the addition of curare $50 \mathrm{mg} / \mathrm{kg}$ intravenously. The serum was injected intravenously or close intra-arterially in single doses ranging from $0 \cdot 1-1 \mathrm{ml}$.

In-vitro tests (I) Isolated rat phrenic nervediaphragm preparation. ${ }^{6}$ The effect of adding serum and plasma obtained at plasmopheresis was tested. In one series of experiments twitch tension elicited by supramaximal stimulation of the phrenic nerve at $0 \cdot 1 \mathrm{~Hz}$ was monitored. In another series twitches were induced by direct muscle stimulation. (II) Isolated innervated chick biventer cervicis muscle preparation. ${ }^{7}$ The effect of serum on twitch responses elicited by supramaximal nerve stimulation was tested.

The preparations were suspended in tissue organ baths containing $25 \mathrm{ml}$ of Krebs-Hanseleit solution for the diaphragm or McEwen's solution for the biventer cervicis muscle. The solutions were aerated with $5 \% \mathrm{CO}_{2}$ in oxygen and maintained at $37^{\circ} \mathrm{C}$ for the diaphragm or $39^{\circ} \mathrm{C}$ for the biventer cervicis muscle preparation. The serum was added in single doses of $0 \cdot 1$, $0 \cdot 2,0.4 \mathrm{ml}$ with washout between, or as the same dose in cumulative mode with no washout. In either case each dose was left in the organ bath for five minutes. In the biventer cervicis preparation the test series was repeated after addition of curare $2 \mathrm{ug} / \mathrm{ml}$ to the solution to produce partial neuro-muscular block. Control preparations were run in parallel.

\section{Results}

Most of the results presented are for thenar muscles. The CAPa to single shocks measured at intervals over several months was low: $2 \cdot 6 \pm 0 \cdot 6$ $\mathrm{mV}$ (mean $\pm \mathrm{SD}$ ), $\mathrm{n}=15$. The values ranged from $2 \cdot 1-3.5 \mathrm{mV}$, with our normal range being $4 \cdot 1$ $21 \cdot 1 \mathrm{mV}$.

Stimulation at $1 \mathrm{~Hz}$ resulted in an exponential decay in CAPa (fig 1). At faster rates of stimulation up to $10 \mathrm{~Hz}$, the response was characterised

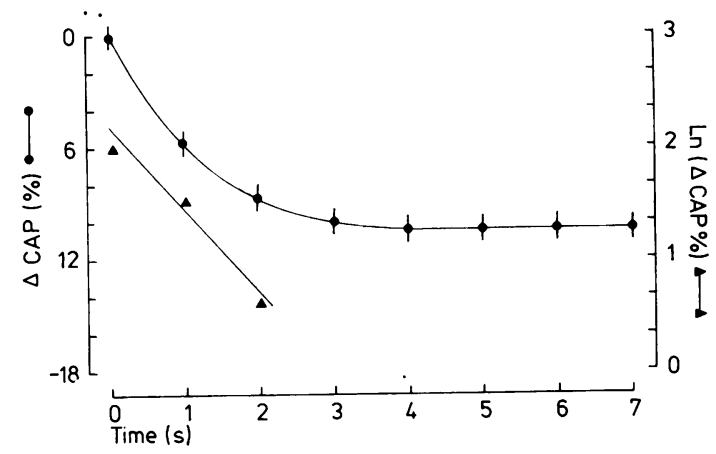

Fig 1 Decay in CAPa, stimulating at $1 \mathrm{~Hz}$. The graph shows mean $\pm S E M, n=8$. The log relationship is shown for the first 3 mean readings. Lines are fitted by eye. Decay time constant is $1.4 \mathrm{~s}$.

by depression followed by facilitation. The extent of depression and facilitation, and the transition point fluctuated in one muscle with time (fig 2A). It also varied between muscles tested on the same occasion (fig 2B). The dual characteristic of transmission was also defined by using twin stimuli (fig 3).

Stimulation at $20 \mathrm{~Hz}$ resulted in facilitation only. Faster rates of stimulation produced no additional potentiation. Fatigue of neuromuscular transmission was tested by prolonged stimulation at $20 \mathrm{~Hz}$. The CAPa reached a plateau after $10 \mathrm{~s}$, and was then maintained for $30 \mathrm{~s}$, dropping thereafter $8 \%$ every $10 \mathrm{~s}$. Normal subjects showed no change in CAPa over a $45 \mathrm{~s}$ period of nerve stimulation at this rate. Facilitation was also observed under physiological circumstances, such as maximal voluntary contraction. The potentiation and its time rate of decay are shown in fig 4.

Guanidine $\mathrm{HCl}$ treatment resulted in a threefold increase in CAPa to $7 \cdot 8 \pm 1 \cdot 1 \mathrm{mV}$ (mean \pm $\mathrm{SD}$ ), range $6.7-9.2 \mathrm{mV}$. In association with this increase in CAPa, facilitation was no longer evident following exercise or during $20 \mathrm{~Hz}$ stimulation. An unexpected feature was noted after the first three days of guanidine treatment. The electrophysiological features of MYS were corrected in thenar muscles, but were still evident in the extensor digitorum brevis muscle (fig 5). After a further four days of treatment the defect was not apparent in either muscle group. The other drugs were administered when the patient was not receiving guanidine.

Edrophonium $\mathrm{HCl}$ infusion resulted in a $30 \%$ increase in CAPa. After one week of oral choline administration CAPa was $2 \cdot 3 \mathrm{mV}$, and after a 


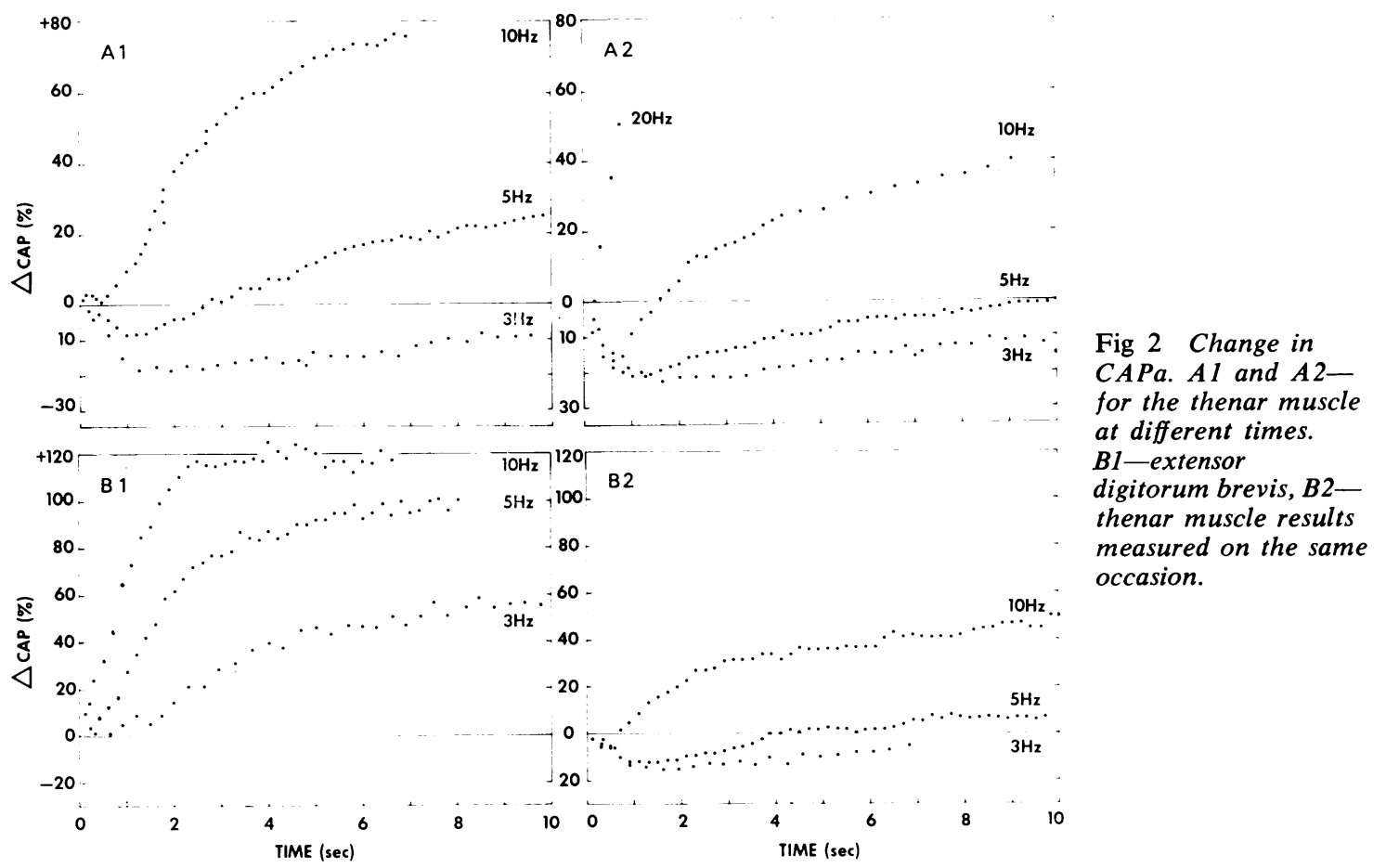

further $2 \frac{1}{2}$ weeks reached $4.5 \mathrm{mV}$. Choline was discontinued and three weeks later CAPa was $3 \cdot 2$ $\mathrm{mV}$ (fig 6A). $20 \mathrm{~Hz}$ stimulation at the corresponding times resulted in potentiation which was similar for the first second, and then diverged (fig 6B). Intravenous administration of choline produced no change in CAPa. The infusion was terminated because the patient complained of lightheadedness, and exhibited flushing of the cheeks, tremor and marked sweating. These features subsided within minutes of cessation of the infusion. For the next 24 hours, the patient experienced some abdominal cramps and diarrhoea. There was no subjective or objective change in strength as a result of guanidine or choline administration.

Pimozide produced no change in CAPa. Plasmapheresis did not result in clinical or electro-

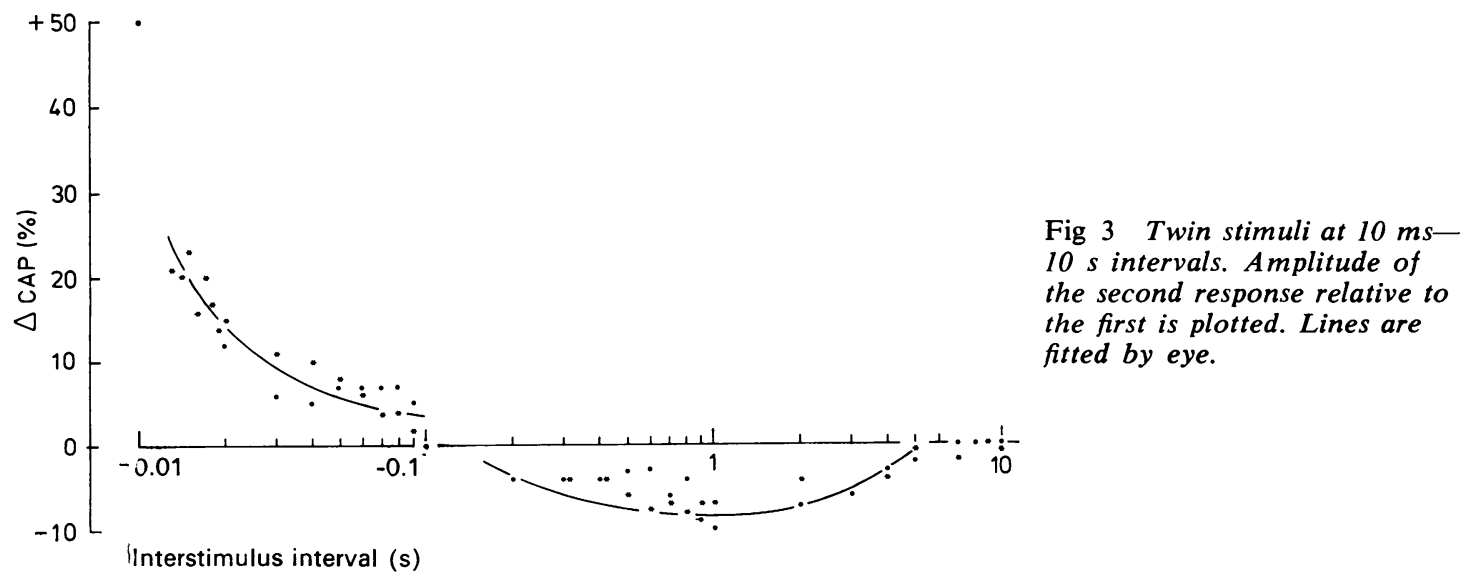




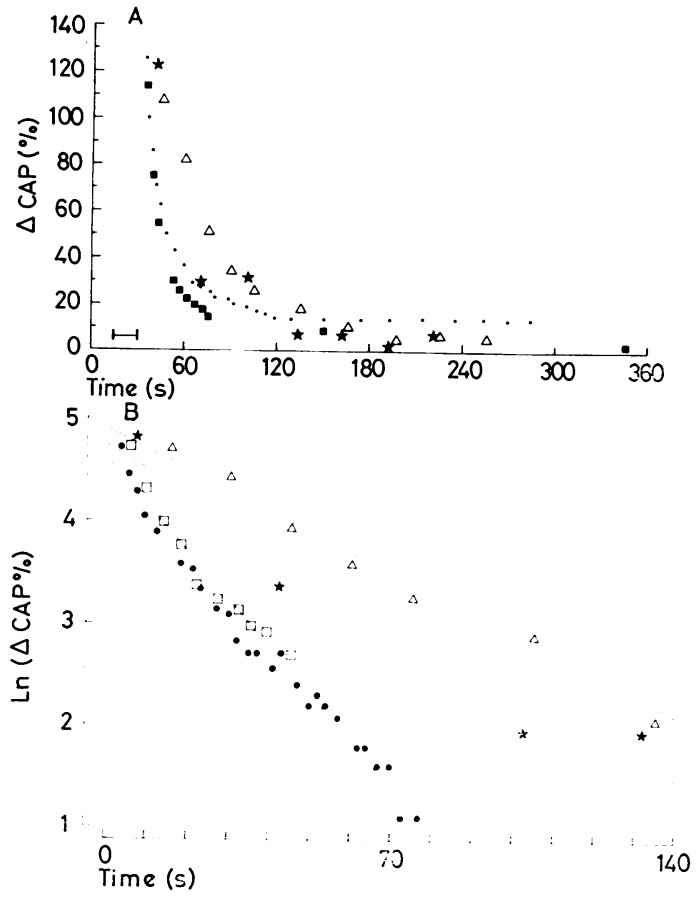

Fig $4 A$ and $B$ : bar represents $15 \mathrm{~s}$ periol of maximal voluntary contraction of thenar muscles. $A$-post-exercise potentiation and its decay, on four occasions, two being on the same day. $B$-results of $A$ plotted on logarithmic scale. Circles and squares values obtained on same day, other values at different times. Time constant for faster decay was $23 \mathrm{~s}$, for slower $51 \mathrm{~s}$. Lines fitted by method of least squares.

physiological improvement over a five month period of observation. The cat preparation and the in-vitro tests showed no change in response as a result of adding the patient's serum.

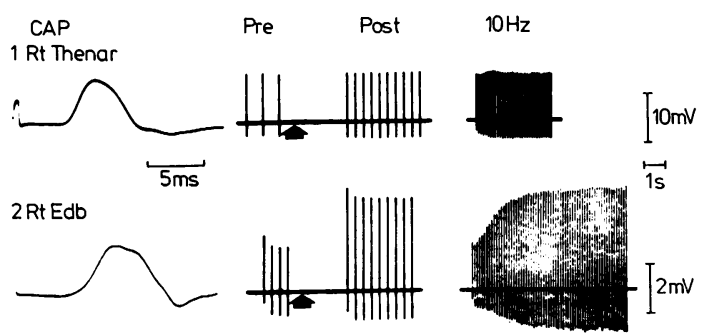

Fig 5 Results after 3 days treatment with guani 'ine. 1-Thenar muscle results normal, 2-extensor digitorum brevis still shows features af $M Y S$.

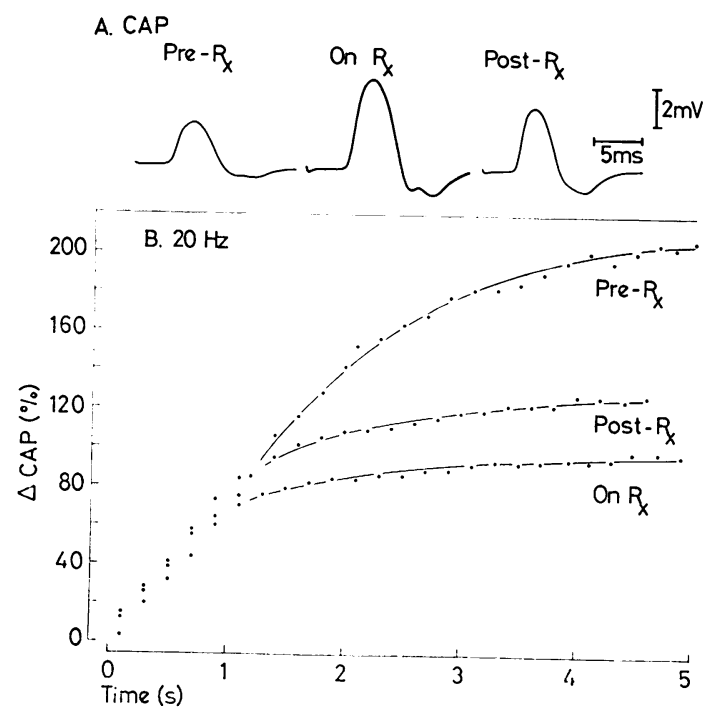

Fig $6 A-C A P a$ at different times in relation to treatment with oral choline $\mathrm{Cl}$. B-change in $\mathrm{CAPa}$ following $20 \mathrm{~Hz}$ stimulation on the same occasions as readings in $A$ were obtained.

\section{Discussion}

MYS is considered to be a pre-junction disorder, due to defect of transmitter release. ${ }^{8}$ The transmission defect has two characteristics-depression and facilitation, depending upon the rate of stimulation. Transmission characteristics fluctuate with time, and can vary with the muscle group examined. The selective effect of guanidine early in the course of treatment further points to the different severity of involvement of muscles in MYS. In-vitro studies have shown that the potentiating effect of guanidine is inversely related to initial quantal content at the neuro-muscular junction. ${ }^{9}$

These findings may be relevant to the reports of patients who apparently have two neuromuscular junction disorders, MG and MYS, concurrently. These reports take two forms. The patient may exhibit electrophysiological features of MG and MYS in different muscle groups at the one time. ${ }^{23}$ Alternatively, the electrophysiological features may fluctuate between those of MG and MYS with time. ${ }^{4}$ These reports were made before estimation of anti-acetylcholine receptor antibody was available. There was also no information on force developed in the affected muscles. This may have been useful as the generation of force is different in MG and MYS. ${ }^{1}$ Our patient's force records were typical of those 
seen in MYS (unpublished observations). An alternative explanation to the twin diagnosis of MG and MYS, may be that the observations represent fluctuation in the dual transmission characteristics of MYS.

The application of quantitative analysis to the electrophysiological changes in MYS has two aims. It defines in more rigorous terms the transmission characteristics, and the way these fluctuate in time. It could be used to compare the nature and extent of the defect between patients. The other potential benefit is that an analysis of the transmission defect may provide a clue to its mechanism. This relates to physiological studies aimed at determining the processes which act to increase transmitter release during and following repetitive stimulation. These have been separated to an extent by their decay time constant. ${ }^{10}$ Of the three processes arbitrarily subdivided on this criterion, our results fit the process of potentiation which decays with a time constant of tens of seconds to minutes. The variability in the decay constant shown in fig 4 , also documents the fluctuation in transmission characteristics which occurs with time. The cause of this variability is uncertain. One possibility is that some change in the factor responsible for the pathophysiology of MYS is occurring. Another potential mechanism is change in local temperature. Animal studies have documented the sensitivity of facilitation of transmitter release to temperature, with the decay time constant of facilitation being inversely related to temperature. ${ }^{11}$ In MYS it has been found that cooling improves transmitter release ${ }^{12}$ and similarly in MG transmission is better at lower temperatures. ${ }^{13}$

The potentiating effect of edrophonium $\mathrm{HCl}$ has been noted previously. ${ }^{14}$ Pimozide was used because of recent evidence indicating the presence of dopamine receptors with inhibitory influence on acetylcholine release at motor nerve terminals. ${ }^{15}$ Pimozide reversed this inhibition. The failure of pimozide to influence the electrophysiological features in this patient argues against the involvement of these receptors in the pathophysiology of MYS.

Choline has been used therapeutically in conditions such as tardive dyskinesia ${ }^{18}$ with the aim of increasing central acetylcholine ( $\mathrm{ACh}$ ) levels. Choline is a precursor of $\mathrm{ACh}$, and its administration in animals has been shown to increase brain ACh concentration. ${ }^{17}$ Direct evidence of functional influence on ACh release is lacking in man, and only indirect evidence is available from animal studies. ${ }^{18}$ In MYS transmitter release at most neuro-muscular junctions is below the normal safety factor. A change in $\mathrm{ACh}$ release as a result of choline administration should therefore be observable. Our results suggest that prolonged oral choline administration caused at least a $40 \%$ increase in the release of an immediately available ACh pool. After 1s of $20 \mathrm{~Hz}$ stimulation, transmission tended to the same value as without treatment. The level of estimated increase in $\mathrm{ACh}$ release is in agreement with histochemical studies in animals showing raised ACh concentration after choline ingestion. ${ }^{17}$ The findings also suggest the presence of at least two ACh pools, only one of which is significantly influenced by choline intake. The existence of separate $\mathrm{ACh}$ pools in nerve terminals has been suggested by animal studies using labelled choline. ${ }^{19}$ It will be of interest to determine whether other patients with MYS show a similar response to oral choline.

Acute choline administration appeared to have no effect on transmitter release at the neuromuscular junction. The clinical features exhibited by the patient were consistent with increased cholinergic autonomic activity. Transmission was therefore increased at some cholinergic sites. The mechanism underlying the difference in effect produced by acute and chronic choline administration, and in the acute setting the disparity in autonomic and neuro-muscular junction response remains uncertain. Animal studies indicate that the half-life of intravenously administered choline is less than one minute. The liver and kidneys remove $50 \%$. Though conversion to ACh is rapid, it was concluded that this was a relatively minor pathway for choline metabolism. ${ }^{20}$ This coupled with the presence of specific high affinity transport systems for choline $^{21}$ in parts of the nervous system suggests that in the acute setting available choline will be selectively distributed. The response to intravenous choline raises the possibility of acute manipulation of cholinergic function, in autonomic and possibly other parts of the nervous system.

Plasmapheresis was without effect. If the MYS was related to the presence of a circulating antibody, as in MG, then the schedule of plasmapheresis used should have produced a beneficial effect. ${ }^{22}$ If it was dependent $\mathrm{n}$ a circulating factor other than an antibody improvement may still have been expected. Alternatively the postulated factor, as the polypeptide botulism toxin, could be tightly bound to its site of action and produce long lasting effects. This is supported by the finding that prolonged washing of 
biopsied inter-costal muscle preparation from patients with MYS fails to alter the transmission block. ${ }^{23}$ The lack of improvement in our patient over a long follow-up period argues against this possibility.

The animal and in-vitro studies showed no evidence to suggest the presence of a circulating factor which impairs neuro-muscular transmission. Fractionation or concentration of the serum was not performed, and it is possible that the methods used were too insensitive or in some other way inappropriate.

\section{References}

1 Lambert EH, Rooke ED. Myasthenic state and lung cancer. In: Lord Brain, Norris FH, eds. The remote effects of cancer on the nervous system. New York: Grune and Stratton, 1965; 67-80.

2 Moir M, Takamori M. Hyperthyroidism and myasthenia gravis with features of EatonLambert Syndrome. Neurol (Minneap) 1976; 26: 882-7.

3 Schwartz MJ, Stalberg E. Myasthenia gravis with features of the myasthenia syndrome. Neurol (Minneap) 1975; 25:80-4.

4 Takamori M, Gutman L. Intermittent defect of acetylcholine release in myasthenia grains. Neurol (Minneap) 1971; 21:47-54.

5 Brown GL. Close arterial injection in cat. $J$ Physiol 1938; 92:22.

6 Bulbring E. Isolated phrenic nerve diaphragm preparation of the rat. Br J Pharmacol 1946; 1: 38-61.

7 Ginsborg BL, Warriner J. Isolated chick biventer cervicis nerve-muscle preparation. Br J Pharmacol 1960; 15:410-11.

8 Elmqvist D, Lambert EH. Detailed analysis of neuromuscular transmission in a patient with the myasthenic syndrome sometimes associated with bronchogenic carcinoma. Mayo Clin Proc 1968; 43:689-713.

9 Kamenskaya MA, Elmqvist D, Thesleff S. Guanidine and neuromuscular transmission. Arch Neurol 1975; 32:505-9.

10 Magleby KL, Zengel JE. Stimulation-induced factors which affect augmentation and potentiation of transmitter release of the neuromuscular junction. J Physiol 1976; 260:687-717.
11 Belnave RJ, Gage PW. Temperature sensitivity of the time course of transmitter release. Brain Res 1970; 21:297-300.

12 Ricker K, Hertel G, Stodieck S. The influence of local cooling on neuromuscular transmission in the myasthenic syndrome of Eaton and Lambert. J Neurol 1977; 217:95-102.

13 Borenstein S, Desmedt JE. Treatment and weather correlates of myasthenic fatigue. Lancet 1974; ii:63-6.

14 Lambert EH. Defects of neuromuscular transmission in syndromes other than myasthenia gravis. Ann NY Acad Sci 1966; 135:367-84.

15 Ganguly DK, Das M. Effects of oxotremorine demonstrate presynaptic muscarinic and dopaminergic receptors on motor nerve terminals. Nature 1979; 278:645-6.

16 Growdon JH, Hirsh MJ, Wurtman RJ, et al. Oral choline administration to patients with tardive dyskinesia. $N$ Engl J Med 1977; 297: 524-7.

17 Cohen EL, Wurtman RJ. Brain acetylcholine control by dietary choline. Science 1976; 191: 561-2.

18 Ulus IH, Wurtman RJ. Choline administration: activation of tyrosine hydroxylase in dopaminergic neurons of rat brain. Science 1975; 194: 1060-1.

19 Aquilonius SM, Fleutge F, Schubert J, Sparf B, Sundival A. Synthesis of acetylcholine in different compartments of brain nerve terminals in vivo as studied by the incorporation of choline from plasma and the effect of pentobarbital on the process. J Neurochem 1973; 20:1509-21.

20 Haubrich DR, Wang PGL, Wedeking PW. Distribution and metabolism of intravenously administered chole (methyl-3H) and synthesis in vivo of acetylcholine in various tissues of guinea pigs. J Pharmacol 1975; 193:246-55.

21 Kuhar MJ, Sethy VH, Roth RT, Aghajanian GK. Choline: selective accumulation by central cholinergic neurons. J Neurochem 1973; 20: 581-93.

22 Dau P, Lindstrom JM, Cassel CK, Denys EH, Shev EE, Spitler LE. Plasmaphoresis and immunosuppressive drug therapy in myasthenia gravis. $N$ Engl J Med 1977; 297:1134-40.

23 Lambert EH, Elmqvist D. Quantal components of end-plate potentials in the myasthenic syndrome. Ann NY Acad Sci 1971; 183:183-99. 